\title{
A GENERAL RELATIVISTIC ROTATING EVOLUTIONARY UNIVERSE
}

\author{
Marcelo Samuel Berman ${ }^{1}$ \\ ${ }^{1}$ Instituto Albert Einstein / Latinamerica \\ Av. Candido Hartmann, 575 - \#17 and \\ 80730-440 - Curitiba - PR - Brazil email: msberman@alberteinsteininstitute.org
}

(Dated: (original: 10 January, 2007; revised: 24 February, 2008).)

\begin{abstract}
We show that when we work with coordinate cosmic time, which is not proper time, RobertsonWalker's metric, includes a possible rotational state of the Universe. An exact formula for the angular speed and the temporal metric coefficient, is found.
\end{abstract}




\section{A GENERAL RELATIVISTIC ROTATING EVO- LUTIONARY UNIVERSE MARCELO SAMUEL BERMAN}

Standard textbooks on Relativistic Cosmology, consider the usual Robertson-Walker's metric, which would represent a homogeneous and isotropic expanding Universe. However, the absence of rotation in the model, cast doubts on its validity as representing a real Universe: it would require a rigorous fine tuning, in order to keep, since inception, a nonrotating Universe. Since the Universe has been observed to be expanding with acceleration, the presence of a positive cosmological constant $(\Lambda)$, has been stressed in the last few years.

As a boundary condition, to be satisfied by Einstein's equations, the Machian condition has been put forward, sometimes in disguise. Berman (2007; 2007a; 2007b) has suggested that the consideration of a zero-total-energy Universe, might represent the Machian desired properties. Brans and Dicke (1961), presented new field equations that would satisfy the approximate relation,

$$
\frac{G M}{c^{2} R} \sim 1
$$

In the above, $G$ represents the gravitational constant, and the mass $M$ is that of a causally related Universe with radius $R$. Sabbata and Sivaran (1994), have shown that a closely related approximation would apply for the spin of the Universe $L$, namely,

$$
\frac{L^{2}}{c^{2} M^{2} R^{2}} \sim 1
$$

We see that the Universe was expected to have a non-zero spin $L$. Though both relations above are heuristic, Berman has shown in the cited references, that the zero-total energy from the Newtonian point of view, could yield several exact relations which substitute the above two approximations. In conclusion: we expect a non-zero spin of the Machian Universe.

As we shall show below, by a simple expedient, we can obtain a rotational and expanding model, out of the original Robertson-Walker's metric. 
Gomide and Uehara (1981) derived the field equations for a Robertson-Walker's metric in terms of coordinate time $(t)$, when this time is not proper time $(\tau)$. In the most simplest case, we may write:

$$
d \tau=\left(g_{00}\right)^{1 / 2} d t
$$

where,

$$
g_{00}=g_{00}(t)
$$

The line element becomes:

$$
d s^{2}=-\frac{R^{2}(t)}{\left(1+k r^{2} / 4\right)^{2}}\left[d \sigma^{2}\right]+g_{00}(t) d t^{2}
$$

The field equations, in General Relativity Theory (GRT) become:

$$
3 \dot{R}^{2}=\frac{1}{3} \kappa\left(\rho+\frac{\Lambda}{\kappa}\right) g_{00} R^{2}-3 k g_{00}
$$

and,

$$
6 \ddot{R}=-g_{00} \kappa\left(\rho+3 p-2 \frac{\Lambda}{\kappa}\right) R-3 g_{00} \dot{R} \dot{g}^{00}
$$

Local inertial processes are observed through proper time, so that the four-force is given by:

$$
F^{\alpha}=\frac{d}{d \tau}\left(m u^{\alpha}\right)=m g^{00} \ddot{x}^{\alpha}-\frac{1}{2} m \dot{x}^{\alpha}\left[\frac{\dot{g}_{00}}{g_{00}^{2}}\right]
$$

Of course, when $g_{00}=1$, the above equations reproduce conventional RobertsonWalker's field equations.

We must mention that the idea behind Robertson-Walker's metric is the Gaussian coordinate system. Though the condition $g_{00}=1$ is usually adopted, we must remember that, the resulting time-coordinate is meant as representing proper time. If we want to use another coordinate time, we still keep the Gaussian coordinate properties.

From the energy-momentum conservation equation, in the case of a uniform Universe, we must have,

$$
\frac{\partial}{\partial x^{i}}(\rho)=\frac{\partial}{\partial x^{i}}(p)=\frac{\partial}{\partial x^{i}}\left(g_{00}\right)=0 \quad(i=1,2,3)
$$


The above is necessary in the determination of cosmic time, for a commoving observer. We can see that the hypothesis (2) - that $g_{00}$ is only time-varying - is now validated.

In order to understand equation (6), it is convenient to relate the rest-mass $m$, with an inertial mass $M_{i}$, with:

$$
M_{i}=\frac{m}{g_{00}} \quad \text {. }
$$

It can be seen that $M_{i}$ represents the inertia of a particle, when observed along cosmic time, i.e., coordinate time. In this case, we observe that we have two acceleration terms, which we call,

$$
a_{1}^{\alpha}=\ddot{x}^{\alpha}
$$

and,

$$
a_{2}^{\alpha}=-\frac{1}{2 g_{00}}\left(\dot{x}^{\alpha} \dot{g}_{00}\right)
$$

The first acceleration is linear; the second, resembles rotational motion.

If we consider $a_{2}^{\alpha}$ a centripetal acceleration, we conclude that the angular speed $\omega$ is given by,

$$
\omega=\frac{1}{2}\left(\frac{\dot{g}_{00}}{g_{00}}\right)
$$

By comparison between the usual $\tau$-metric, and the field equations in the $t$-metric, we are led to conclude that the conventional energy density $\rho$ and cosmic pressure $p$ are transformed into $\bar{\rho}$ and $\bar{p}$, where:

$$
\bar{\rho}=g_{00}\left(\rho+\frac{\bar{\Lambda}}{\kappa}\right)
$$

and,

$$
\bar{p}=g_{00}\left(p-\frac{\bar{\Lambda}}{\kappa}\right)
$$

We plug back into the field equations, and find, 


$$
\bar{\Lambda}=\Lambda-\frac{3}{2 \kappa}\left(\frac{\dot{R}}{R}\right) \dot{g}^{00}
$$

For a time-varying angular speed, considering an $\operatorname{arc} \phi$, so that,

$$
\omega(t)=\frac{d \phi}{d t}=\dot{\phi}
$$

we find, from (11),

$$
g_{00}=C e^{2 \phi(t)} \quad \cdot \quad(C=\text { constant })
$$

Returning to (14), we find,

$$
\bar{\Lambda}=\Lambda+\frac{3}{\kappa C}\left(\frac{\dot{R}}{R}\right) \omega e^{-2 \phi(t)} \quad .
$$

We see that there is no singularity in the above relations for $g_{00}$ and $\bar{\Lambda}$.

We have found an exact singularity-free solution, in GRT, for a rotating evolutionary Universe, derived from the original Robertson-Walker's metric, endowed with a lambdaterm. The second right-hand-side term in (17) can be neglected as time passes by. The inertial mass $M_{i}$, depends on the rotation of the expanding Universe, fulfilling Mach's Principle.

The $\tau$-field equations represent the compass of inertia; the $t$-metric, represents the rotation relative to the first one. By solving the field equations, one may find the cosmic pressure and energy density, for a given equation of state.

Gödel's Universe (Adler et al., 1975), in which the matter does not uniquely determine the geometry, violates Mach's Principle, so that, there is not a distinguished universal timecoordinate in such Universe. More than that, the bulk matter of that Universe is commoving relative to a particular reference frame, but this frame is not inertial. The compass of inertia should rotate relative to the matter, or vice-versa, but the bulk matter represents the "fixed starts", so that, according to Mach's Principle, both can not rotate one relative to the other.

This is not the case in the present model. The background $\tau$ - metric defines the "fixed stars" . The rotation becomes evident in the $t$-metric. All observers are commoving. This is done in a singularity-free framework. 
The rotation of the Universe is a subject dealt by Berman (2007, 2007a, 2007b).

\section{Acknowledgments}

The chief editor of this journal, has contributed with suggestions, towards the clarification of the material in this paper. To him, the author expresses his recognition, and also to his intellectual mentors, now friends and colleagues, M.M. Som and F.M. Gomide. He thanks the many other colleagues that collaborate with him. The typing was made by Marcelo F. Guimarães, who I consider a friend and to whom my thanks are due for this and many other collaborations. I am grateful for the support by Geni, Albert and Paula.

\section{References}

Adler, R.J.; Bazin, M.; Schiffer, M. (1975) - Introduction to General Relativity, Second Edition, McGraw-Hill, New York.

Berman,M.S. (2007) - Introduction to General Relativity, and the Cosmological Constant Problem, Nova Science, New York.

Berman,M.S. (2007a) - Introduction to General Relativistic and Scalar-Tensor Cosmologies, Nova Science, New York.

Berman,M.S. (2007b) - The Pioneer Anomaly and a Machian Universe, Astrophysics and Space Science, 312, 275.

Brans, C.; Dicke, R.H. (1961) - Physical Review, 124, 925.

Gomide, F.M.; Uehara, M. (1981) - Astron. Astrophys., 95, 362.

Sabbata, V. de; Sivaran, C. (1994) - Spin and Torsion in Gravitation, World Scientific, Singapore. 